PROCEEDINGS OF THE

AMERICAN MATHEMATICAL SOCIETY

Volume 132, Number 3, Pages 659-667

S 0002-9939(03)07134-X

Article electronically published on July 31, 2003

\title{
ON INVARIABILITY OF SELF-INJECTIVE ALGEBRAS OF TILTED TYPE UNDER STABLE EQUIVALENCES
}

\author{
ANDRZEJ SKOWROŃSKI AND KUNIO YAMAGATA \\ (Communicated by Martin Lorenz)
}

\begin{abstract}
We prove that a large class of self-injective algebras of tilted type is invariant under stable equivalences of module categories.
\end{abstract}

\section{IntRoduCtion AND THE MAIN RESUlT}

Throughout the paper, by an algebra is meant a basic, connected, Artin algebra (associative, with an identity) over a fixed commutative Artinian ring $K$. For an algebra $A$, we denote by $\bmod A$ the category of finitely generated right $A$-modules and by $\underline{\bmod } A$ the stable module category of $A$. Recall that the objects of $\underline{\bmod } A$ are the objects of $\bmod A$ without nonzero projective direct summands, and for any two objects $M$ and $N$ in $\underline{\bmod } A$ the $K$-module $\underline{\operatorname{Hom}}_{A}(M, N)$ of morphisms from $M$ to $N$ is the quotient $\operatorname{Hom}_{A}(M, N) / P(M, N)$, where $P(M, N)$ is the submodule of $\operatorname{Hom}_{A}(M, N)$ consisting of all $A$-homomorphisms that factorize through projective $A$-modules. Two algebras $A$ and $\Lambda$ are said to be stably equivalent if their stable module categories $\underline{\bmod } A$ and $\underline{\bmod } \Lambda$ are equivalent. Recall also that $D=\operatorname{Hom}_{K}(-, E)$, where $E$ is a minimal injective cogenerator in $\bmod K$, defines a duality between the categories of left and right modules. An algebra $A$ is called self-injective if $A \cong D(A)$ in $\bmod A$, that is, the projective $A$-modules are injective. An important class of self-injective algebras is formed by the algebras of the form $\widehat{B} / G$ where $\widehat{B}$ is the repetitive algebra [5] (locally bounded, without identity)

$$
\widehat{B}=\bigoplus_{k \in \mathbb{Z}}\left(B_{k} \oplus(D B)_{k}\right)
$$

of an algebra $B$, where $B_{k}=B$ and $(D B)_{k}=D B$ for all $k \in \mathbb{Z}$, the multiplication in $\widehat{B}$ is defined by

$$
\left(a_{k}, f_{k}\right)_{k} \cdot\left(b_{k}, g_{k}\right)_{k}=\left(a_{k} b_{k}, a_{k} g_{k}+f_{k} b_{k+1}\right)_{k \in \mathbb{Z}}
$$

for $a_{k}, b_{k} \in B_{k}, f_{k}, g_{k} \in(D B)_{k}$, and $G$ is an admissible group of $K$-automorphisms of $\widehat{B}$. More precisely, for a fixed set $\mathcal{E}=\left\{e_{i} \mid 1 \leq i \leq m\right\}$ of primitive orthogonal idempotents of $B$ with $1_{B}=e_{1}+\cdots+e_{m}$, consider the canonical set $\widehat{\mathcal{E}}=\left\{e_{j, k} \mid 1 \leq\right.$ $j \leq m, k \in \mathbb{Z}\}$ of primitive orthogonal idempotents of $\widehat{B}$ such that $e_{j, k} \widehat{B}=\left(e_{j} B\right)_{k} \oplus$ $\left(e_{j} D B\right)_{k}$ for $1 \leq j \leq m$ and $k \in \mathbb{Z}$. By an automorphism of $\widehat{B}$ we mean a $K$-algebra

Received by the editors July 10, 2002 and, in revised form, October 28, 2002.

2000 Mathematics Subject Classification. Primary 16D50, 16G10, 16G70, 16S70.

Key words and phrases. Self-injective algebra, tilted algebra, stable equivalence. 
automorphism of $\widehat{B}$ that fixes the chosen set $\widehat{\mathcal{E}}$ of primitive orthogonal idempotents of $\widehat{B}$. A group $G$ of automorphisms of $\widehat{B}$ is said to be admissible if the induced action of $G$ on $\widehat{\mathcal{E}}$ is free and has finitely many orbits. Then the orbit algebra $\widehat{B} / G$ is a self-injective algebra and the $G$-orbits in $\widehat{\mathcal{E}}$ form a canonical set of primitive orthogonal idempotents of $\widehat{B} / G$ whose sum is the identity of $\widehat{B} / G$ (see [4]). We denote by $\nu_{\widehat{B}}$ the Nakayama automorphism of $\widehat{B}$ such that $\nu_{\widehat{B}}\left(e_{j, k}\right)=e_{j, k+1}$ for all $1 \leq j \leq m, k \in \mathbb{Z}$. Then the infinite cyclic group $\left(\nu_{\widehat{B}}\right)$ generated by $\nu_{\widehat{B}}$ is admissible and $\widehat{B} /\left(\nu_{\widehat{B}}\right)$ is the trivial extension $B \ltimes D B$ of $B$ by $D B$. An automorphism $\varphi$ of $\widehat{B}$ is said to be positive (respectively, rigid) when $\varphi\left(B_{k}\right) \subseteq \sum_{i \geq k} B_{i}$ (respectively, $\left.\varphi\left(B_{k}\right)=B_{k}\right)$ for any $k \in \mathbb{Z}$. Moreover, $\varphi$ is said to be strictly positive if it is positive but not rigid. We shall also consider $\widehat{B}$ as a locally bounded $K$-category with the objects set as $\widehat{\mathcal{E}}$.

Let $R$ be a tilted algebra of type $\Delta$ that is not a Dynkin quiver. Then the Auslander-Reiten quiver $\Gamma_{\widehat{R}}$ of $\widehat{R}$ is of the form

$$
\Gamma_{\widehat{R}}=\bigvee_{p \in \mathbb{Z}}\left(\mathcal{X}_{p} \vee \mathcal{R}_{p}\right)
$$

where, for each $p \in \mathbb{Z}, \mathcal{X}_{p}$ is a component with the stable part of the form $\mathbb{Z} \Delta, \mathcal{R}_{p}$ is a family of components whose stable parts are tubes (if $\Delta$ is Euclidean) or of type $\mathbb{Z A}_{\infty}$ (if $A$ is wild), and $\nu_{\widehat{R}}\left(\mathcal{X}_{p}\right)=\mathcal{X}_{p+2}$ and $\nu_{\widehat{R}}\left(\mathcal{R}_{p}\right)=\mathcal{R}_{p+2}$, for the induced action of $\nu_{\widehat{R}}$ on $\Gamma_{\widehat{R}}$ (see [1], 3], [6]). Furthermore, an automorphism $\varphi$ of $\widehat{R}$ is positive (respectively, strictly positive) if and only if there exists $q \geq 0$ (respectively, $q>0$ ) such that $\varphi\left(\mathcal{X}_{p}\right)=\mathcal{X}_{p+q}$ and $\varphi\left(\mathcal{R}_{p}\right)=\mathcal{R}_{p+q}$ for all $p \in \mathbb{Z}$. We also note that the class of algebras of the form $\widehat{R} /\left(\psi \nu_{\widehat{R}}\right)$ with $R$ a tilted algebra of type $\Delta$ (not a Dynkin quiver) and $\psi$ a strictly positive (respectively, positive) automorphism of $\widehat{R}$ coincides in the class of all self-injective algebras of tilted type whose stable Auslander-Reiten quiver admits at least three (respectively, two) components of type $\mathbb{Z} \Delta$. We have proved in $[8$, Theorem 1$]$ that a self-injective algebra is stably equivalent to an algebra $\widehat{R} /\left(\psi \nu_{\widehat{R}}\right)$ with $\psi$ a positive automorphism of $\widehat{R}$ if and only if $A$ is a socle equivalent to an algebra $\widehat{B} /\left(\varphi \nu_{\widehat{B}}\right)$ where $B$ is a tilted algebra of type $\Delta$ and $\varphi$ is a positive automorphism of $\widehat{B}$. Recall that two self-injective algebras $A$ and $\Lambda$ are called socle equivalent if the factor algebras $A / \operatorname{soc} A$ and $\Lambda / \operatorname{soc} \Lambda$ are isomorphic. Our main result shows that for $\psi$ strictly positive we may replace "socle equivalent" by "isomorphic".

Theorem. Let $A$ be a self-injective algebra stably equivalent to a self-injective algebra of the form $\widehat{R} /\left(\psi \nu_{\widehat{R}}\right)$ where $R$ is a tilted algebra of type $\Delta$ that is not a Dynkin quiver and $\psi$ is a strictly positive automorphism of $\widehat{R}$. Then $A$ is isomorphic to an algebra of the form $\widehat{B} /\left(\varphi \nu_{\widehat{B}}\right)$ for some tilted algebra $B$ of type $\Delta$ and a strictly positive automorphism $\varphi$ of $\widehat{B}$.

We note that the strict positivity of $\psi$ is necessary for the validity of the theorem. Namely, if $A$ is a nonsplittable Hochschild extension of a hereditary algebra $H$ not of Dynkin type (see 77 and [10] for existence of such algebras), then $A$ is stably equivalent to the trivial extension $H \ltimes D(H) \cong \widehat{H} /\left(\nu_{\widehat{H}}\right)$ but is not isomorphic to an algebra $\widehat{B} /\left(\varphi \nu_{\widehat{B}}\right)$ with $B$ a tilted algebra of type $\Delta$ and $\varphi$ a positive automorphism 
of $\widehat{B}$. It would be interesting to know if the theorem also holds for self-injective algebras of Dynkin type.

For general background concerning representation theory of Artin algebras and self-injective algebras we refer to [2] and [1].

\section{Preliminary Results}

The aim of this section is to prove some preliminary results that we need in the proof of our main theorem. As an application we establish also a criterion (Proposition 2.6) for a self-injective Artin algebra $A$ to be isomorphic to an algebra of the form $\widehat{B} / G$, where $\widehat{B}$ is the repetitive algebra of an Artin algebra $B$ of finite

global dimension and $G$ is an admissible infinite cyclic group of automorphisms of $\widehat{B}$.

Let $A$ be a self-injective algebra, $\left\{e_{i} \mid 1 \leq i \leq n\right\}$ a fixed set of primitive orthogonal idempotents of $A$ such that $1_{A}=e_{1}+\cdots+e_{n}$, and $\nu=\nu_{A}$ a fixed Nakayama automorphism of $A$ inducing an $A$-bimodule isomorphism $A \cong{ }_{\nu} D A$, where ${ }_{\nu} D A$ denotes the left $A$-module obtained from the canonical left $A$-module $D A$ by changing the operation of $A$ by $a \cdot f=\nu(a) f$ for $a \in A, f \in D A$. Hence we have $\operatorname{soc}\left(e_{i} A\right) \cong \operatorname{top}\left(\nu\left(e_{i}\right) A\right)\left(=\nu\left(e_{i}\right) A / \nu\left(e_{i}\right) \operatorname{rad} A\right)$ as right $A$-modules for all $i \in\{1, \ldots, n\}$. Since $\left\{\nu\left(e_{i}\right) A \mid 1 \leq i \leq n\right\}$ is a set of representatives of indecomposable projective $A$-modules, there is a permutation of $\{1, \ldots, n\}$, denoted again by $\nu$, such that $\nu\left(e_{i}\right) A \cong e_{\nu(i)} A$ for all $i \in\{1, \ldots, n\}$. Let $I$ be a (two-sided) ideal of $A, B=A / I$ and $e$ an idempotent of $A$ such that $e+I$ is the identity of $B$. We may assume that $e=e_{1}+\cdots+e_{m}$ for some $m \leq n$, and $\left\{e_{i} \mid 1 \leq i \leq m\right\}$ is the subset of $\left\{e_{i} \mid 1 \leq i \leq n\right\}$ consisting of all idempotents $e_{i}$ that are not in $I$. Then such an idempotent $e$ is uniquely determined by $I$ up to an inner automorphism of $A$ and is called a residual identity of $B$. We note that $B \cong e A e / e I e$ and $1-e \in I$.

Let $A$ be a self-injective algebra, $I$ an ideal of $A$, and $B=A / I$. For idempotents $f$ and $f^{\prime}$ of $A, f$ is said to be a summand of $f^{\prime}$ if $f f^{\prime}=f^{\prime} f=f$, and we denote this fact by $f \leq f^{\prime}$. Moreover, the orthogonality $f f^{\prime}=0=f^{\prime} f$ of $f$ and $f^{\prime}$ is denoted by $f \perp f^{\prime}$. Fix a set $\left\{e_{i} \mid 1 \leq i \leq n\right\}$ of primitive orthogonal idempotents of $A$ such that $1_{A}=\sum_{i=1}^{n} e_{i}$ and $e=\sum_{i=1}^{m} e_{i}$, for some $m \leq n$, is a residual identity of $B$. We denote the residue class of each idempotent $e_{j}, 1 \leq j \leq m$, in $B=A / I$ by $e_{j}$ again, for simplicity. Since $B \cong e A e / e I e$ as algebras, we often identify them. Finally, for $i \in\{1, \ldots, m\}$, let $e_{B}^{(i)}$ (respectively, $e_{I}^{(i)}$ ) be the sum of all idempotents $e_{j}, 1 \leq j \leq n$, such that $e_{i} B e_{j} \neq 0$ (respectively, $e_{i}(I / \operatorname{soc} I) e_{j} \neq 0$ ). Following [7] (2.1)] the ideal $I$ is said to be deforming if $e I e=\ell_{e A e}(I)=r_{e A e}(I)$ and the ordinary quiver $Q(B)$ of $B$ has no oriented cycles. Here, $\ell_{e A e}(I)$ is the left annihilator of $I$ in $e A e$ and $r_{e A e}(I)$ is the right annihilator of $I$ in $e A e$.

From now on we assume that the ideal $I$ is deforming. For the proofs of our main results, we need several technical lemmas. We recall first the following properties of a deforming ideal proved in [7, Section 1].

Lemma 2.1. (1) $\operatorname{Soc}(A) \subseteq I$.

(2) For $e_{i}(i \leq m)$, the right $B$-modules $e_{i} B$ and $e_{i}(I / \operatorname{soc} I)$ have no common composition factors, and similarly for $e_{i}(\operatorname{rad} B)$ and $e_{i} I$.

(3) There are no idempotents $e_{i_{j}}(0 \leq j \leq t)$ such that

$$
e_{i_{0}}(\operatorname{rad} B) e_{i_{1}} \neq 0, e_{i_{1}}(\operatorname{rad} B) e_{i_{2}} \neq 0, \ldots, e_{i_{t-1}}(\operatorname{rad} B) e_{i_{t}} \neq 0, e_{i_{t}}(\operatorname{rad} B) e_{\nu\left(i_{0}\right)} \neq 0 .
$$


(4) $e_{i} I e_{B}^{(i)} \subseteq \operatorname{soc}\left(e_{i} I\right)$ and $e_{i} A e_{I}^{(i)} \subseteq e_{i} I$ for $i \leq m$.

(5) $e_{B}^{(i)} \perp e_{I}^{(i)}$.

(6) $e^{(i)} a=e_{i} a e_{B}^{(i)}+e_{i} a e_{I}^{(i)}+e_{i} a e_{\nu(i)}$ for $a \in A$ and $1 \leq i \in m$ with $\nu(i) \neq i$.

Lemma 2.2. For any $i \leq m$, the following equalities hold:

(1) $e_{i}(e A e) e_{I}^{(i)} I=0$;

(2) $e_{i} \operatorname{rad}(e A e) e_{\nu(i)} I=0$ and $I e_{\nu^{-1}(i)} \operatorname{rad}(e A e) e_{i}=0$.

Proof. (1) Assume that $e_{i} a e_{I}^{(i)} I \neq 0$ for some $a \in e A e$. Since $e e_{I}^{(i)} \neq 0$ by assumption, there is some $e_{j} \leq e$ such that $e_{j} \leq e_{I}^{(i)}$ and $e_{i} a e_{j} I \neq 0$. This implies that $e_{i} a e_{j} \notin e I e$, because $\ell_{e A e}(I)=e I e$ by our assumption. Thus $0 \neq e_{i} \bar{a} e_{j} \in B$ so that $e_{j} \leq e_{B}^{(i)}$, where $\bar{a}$ denotes the residue class of $a$ in $A / I$. Then it follows that $e_{B}^{(i)} e_{I}^{(i)} \neq 0$, a contradiction to Lemma 2.1 $(2)$.

(2) Assume that $e_{i} a e_{\nu(i)} I \neq 0$ for some $a=e a e \in \operatorname{rad}(e A e)$. In particular, we have $e e_{\nu(i)} \neq 0$, so that $e_{\nu(i)} \leq e$ and $e_{i} a e_{\nu(i)} \notin e I e$, because $\ell_{e A e}(I)=e I e$. Thus we have $e_{i}(\operatorname{rad} B) e_{\nu(i)} \neq 0$, a contradiction to Lemma 2.1](3). The proof of the second equality is similar.

Lemma 2.3. If $1 \leq i, \nu(i) \leq m$, then $e_{i}(I / \operatorname{soc} I) e_{\nu(i)}=0$ and $e_{i}(\operatorname{rad} A) e_{\nu(i)} \subseteq$ $\operatorname{soc}\left(e_{i} I\right)$.

Proof. (1) If $e_{i}(I / \operatorname{soc} I) e_{\nu(i)} \neq 0$, then $e_{\nu(i)}(\operatorname{rad} B) e_{\nu(i)} \neq 0$ by [7, Lemma 1.2], which implies that $\mathcal{Q}(B)$ has an oriented cycle, a contradiction. Hence we have $e_{i}(I / \operatorname{soc} I) e_{\nu(i)}=0$.

(2) By Lemma 2.11(3), $e_{i}(\operatorname{rad} B) e_{\nu(i)}=0$ and so $e_{i}(\operatorname{rad} A) e_{\nu(i)} \subseteq e_{i} I e_{\nu(i)}$. If $e_{i}(\operatorname{rad} A) e_{\nu(i)} \nsubseteq \operatorname{soc}\left(e_{i} I\right)$, then $e_{i}(\operatorname{rad} A / \operatorname{soc} I) e_{\nu(i)} \neq 0$. Hence, it follows from Lemma 2.1 (2) that $e_{i}(I / \operatorname{soc} I) e_{\nu(i)} \neq 0$, which is impossible by the assertion proved in (1) above. Thus we conclude that $e_{i}(\operatorname{rad} A) e_{\nu(i)} \subseteq \operatorname{soc}\left(e_{i} I\right)$.

Lemma 2.4. For $a, b \in A \backslash I$, the following statements hold:

(1) if $a b \in \operatorname{rad} A$, then $e_{i}$ aebe ${ }_{\nu(i)}=0$ for $1 \leq i, \nu(i) \leq m$;

(2) $e_{i} a_{e b e_{(i)}}=0$ for $1 \leq i, \nu(i) \leq m$ and $\nu(i) \neq i$.

Proof. (1) It suffices to prove the assertion for $a=e_{i} a e_{j}$ and $b=e_{j} b e_{\nu(i)}$ for $e_{j} \in B$. Suppose that $a b \neq 0$. We claim that both $a$ and $b$ belong to $\operatorname{rad} A$. In fact, if $a \notin \operatorname{rad} A$, then $e_{i}=e_{j}$ and there is some $a^{\prime} \in e_{i} A e_{i}$ with $a^{\prime} a=e_{i}$. Then $b=a^{\prime}(a b) \in \operatorname{soc} I$ because $a b \in \operatorname{soc} I$ by Lemma 2.3 a contradiction to the assumption. Thus $a \in \operatorname{rad} A$. Similarly, we have $b \in \operatorname{rad} A$. It therefore follows that $e_{i} B e_{j} \neq 0$ and $e_{j} B e_{\nu(i)} \neq 0$, a contradiction to Lemma 2.1] 3 ). (1).

(2) Since $\nu(i) \neq i, e_{i} a e b e_{\nu(i)} \in \operatorname{rad} A$ obviously, and the assertion follows from

Denote by $\varrho: e A e \longrightarrow e A e / e I e=B$ the canonical algebra epimorphism and define the map $\phi: e A e / e I e \longrightarrow e A e$ by

$$
\phi(a+e I e)=\sum_{i=1}^{m} e_{i} a e_{B}^{(i)}
$$

for all $a \in e A e$.

Proposition 2.5. Assume $\nu(i) \neq i$ for all $i \in\{1, \ldots, m\}$. Then $\phi$ is an algebra homomorphism such that $\varrho \phi=1_{B}$. 
Proof. We first prove that $\phi$ is a well-defined $K$-homomorphism. It suffices to show that $e_{i} I e_{B}^{(i)}=0$ for any $i \leq m$. For this, suppose that $e_{i} I e_{B}^{(i)} \neq 0$. Then $e_{i} I e_{B}^{(i)} e_{\nu(i)} \neq 0$, because $e_{i} I e_{B}^{(i)} \subseteq \operatorname{soc}\left(e_{i} I\right) \cong \operatorname{top}\left(e_{\nu(i)} A\right)$ by Lemma 2.1 (4). In particular, $e_{B}^{(i)} e_{\nu(i)} \neq 0$ and so $e_{\nu(i)} \leq e_{B}^{(i)}$. Hence $e_{i} B e_{\nu(i)} \neq 0$ by definition. On the other hand, $e_{i}(\operatorname{rad} B) e_{\nu(i)}=0$ by Lemma 2.1(3). Thus we have $e_{\nu(i)}=e_{i}$, equivalently $\nu(i)=i$, a contradiction.

The proof that $\phi$ is an algebra homomorphism, is divided into several steps, and involves the relations (5) and (6) of Lemma 2.1.

(1) $\phi(e+e I e)=e$.

For $a=e, e_{i} a e_{I}^{(i)}=e_{i} e_{I}^{(i)}=0$ because $e_{i} \leq e_{B}^{(i)}$ and $e_{B}^{(i)} \perp e_{I}^{(i)}$, and $e_{i} a e_{\nu(i)}=$ $e_{i} e_{\nu(i)}=0$ because $\nu(i) \neq i$. Hence $\phi(e+e I e)=\sum_{i=1}^{m} e_{i} e e_{B}^{(i)}=e$.

(2) $\phi((a+e I e)(b+e I e))=\phi(a+e I e) \phi(b+e I e)$ for $a, b \in e A e$.

We may assume that $a=e_{i} a e_{i^{\prime}}, b=e_{j} b e_{j^{\prime}}$ for $e_{i^{\prime}} \leq e_{B}^{(i)}, e_{j^{\prime}} \leq e_{B}^{(j)}$. Moreover, it is enough to consider the case when $a \notin I$ and $b \notin I$. Let $\alpha=\phi((a+e I e)(b+e I e))$ and $\beta=\phi(a+e I e) \phi(b+e I e)$. Then $\alpha=\phi(a b+e I e)=e_{i} a e_{i^{\prime}} e_{j} b e_{j^{\prime}} e_{B}^{(i)}$, and $\beta=e_{i} a e_{i^{\prime}} e_{B}^{(i)} \cdot e_{j} b e_{j^{\prime}} e_{B}^{(j)}$. Here, if $e_{j} \not \leq e_{B}^{(i)}$, then $e_{B}^{(i)} e_{j}=0$ and so $e_{j^{\prime}} e_{j}=0$. Hence we have $\alpha=0$ and $\beta=0$, and $\alpha=\beta$ as required. Now, assume that $e_{j} \leq e_{B}^{(i)}$. Then $\beta=e_{i} a e_{i^{\prime}} e_{j} b e_{j^{\prime}} e_{B}^{(j)}$. Since $e_{B}^{(i)} e_{B}^{(j)}=e_{B}^{(j)} e_{B}^{(i)}$, it therefore suffices to show that $\alpha=\alpha e_{B}^{(j)}$ and $\beta=\beta e_{B}^{(i)}$.

(a) First we show that $\alpha=\alpha e_{B}^{(j)}$. Obviously, $e_{j} b e_{j^{\prime}} e_{B}^{(i)}=e_{j} b e_{j^{\prime}} e_{B}^{(i)}\left(e_{B}^{(j)}+\right.$ $\left.e_{I}^{(j)}+e_{\nu(j)}\right)$. We claim that $e_{j} b e_{j^{\prime}} e_{B}^{(i)} e_{I}^{(j)}=0$ and $e_{j} b e_{j^{\prime}} e_{B}^{(i)} e_{\nu(j)}=0$. In fact, if $e_{j} b e_{j^{\prime}} e_{B}^{(i)} e_{I}^{(j)} \neq 0$, then $0 \neq e_{j^{\prime}} e_{B}^{(i)} e_{I}^{(j)}=e_{j^{\prime}} \leq e_{I}^{(j)}$. Since $e_{j} b e_{j^{\prime}}=b \notin I$ by assumption, we have $e_{j} B e_{I}^{(j)} \neq 0$, which contradicts the orthogonality $e_{B}^{(j)} \perp$ $e_{I}^{(j)}$. Next, if $e_{j} b e_{j^{\prime}} e_{B}^{(i)} e_{\nu(j)} \neq 0$, then $0 \neq e_{j^{\prime}} e_{B}^{(i)} e_{\nu(j)}=e_{j^{\prime}}=e_{\nu(j)}$ and $b=$ $e_{j} b e_{\nu(j)}$. Hence $e_{j} b e_{\nu(j)} \in \operatorname{rad} A \backslash I$ because $j \neq \nu(j)$ by assumption so that $e_{j}(\operatorname{rad} B) e_{\nu(j)} \neq 0$, which contradicts Lemma 2.1 (3). Thus we have proved that $e_{j} b e_{j^{\prime}} e_{B}^{(i)}=e_{j} b e_{j^{\prime}} e_{B}^{(i)} e_{B}^{(j)}$, so that $\alpha=\alpha e_{B}^{(j)}$.

(b) Secondly, we show that $\beta=\beta e_{B}^{(i)}$. Since $\beta=e_{i} \beta=e_{i} \beta e_{B}^{(i)}+e_{i} \beta\left(e_{I}^{(i)}+\right.$ $\left.e_{\nu(i)}\right)$, it suffices to show that $\beta e_{I}^{(i)}=0$ and $\beta e_{\nu(i)}=0$. Suppose that $\beta e_{I}^{(i)} \neq 0$ contrarily. Then it follows from the proof of [7, Proposition 3.1] that $\beta e_{I}^{(i)} \in$ $\operatorname{soc}\left(e_{i} I\right)$. Hence $\beta e_{I}^{(i)} e_{\nu(i)} \neq 0$, and so $e_{j^{\prime}} e_{B}^{(j)} e_{I}^{(i)} e_{\nu(i)} \neq 0$. It follows that $e_{j^{\prime}} e_{B}^{(j)} e_{I}^{(i)} e_{\nu(i)}=e_{\nu(i)}=e_{j^{\prime}} \leq e$. Therefore, $\beta=e_{i} a e_{j} b e_{\nu(i)}$, so that $\beta=0$ by Lemma $2.4(2)$, a contradiction.

Therefore, we proved that $\phi$ is an algebra homomorphism. Finally, for $a=e a e \in$ $e A e, a-\sum_{i=1}^{m} e_{i} a e_{B}^{(i)}=\sum_{i=1}^{m}\left(e_{i} a e-e_{i} a e_{B}^{(i)}\right)=\sum_{i=1}^{m}\left(e_{i} a e_{I}^{(i)}+e_{i} a e_{\nu(i)}\right)$, which belongs to $e I e$. Hence we have $\varrho \phi=1_{B}$.

We will now prove the announced criterion.

Proposition 2.6. Let $A$ be a self-injective algebra, $I$ an ideal of $A, B=A / I$, and e a residual identity of $B$. Assume that the ordinary quiver $Q(B)$ of $B$ has no oriented cycles, IeI $=0$, Ie is an injective cogenerator in $\bmod B$, and $e_{i} \neq e_{\nu(i)}$ for any $i \in\{1, \ldots, m\}$. Then $A$ is isomorphic to an algebra $\widehat{B} / G$ where $G$ is an 
infinite cyclic group of automorphisms of $\widehat{B}$ generated by $\varphi \nu_{\widehat{B}}$ for some positive automorphism $\varphi$ of $\widehat{B}$.

Proof. It follows from [7, Proposition 2.3] that $I$ is a deforming ideal, and by Proposition [2.5 the canonical epimorphism $\varrho: e A e \longrightarrow e A e / e I e=B$ splits. Applying now [9, Theorem 3.8] we conclude that $A$ is isomorphic to $\widehat{B} /\left(\varphi \nu_{\widehat{B}}\right)$ for some positive automorphism $\varphi$ of $\widehat{B}$.

\section{SELF-InJECTIVE AlgEBRAS WIth DEForming IDEAL}

Let $A$ be a self-injective algebra, $I$ a deforming ideal of $A, B=A / I$ and $e$ a residual identity of $B$. Then $I$ can be considered as a $(e A e / e I e)$-bimodule. Denote by $A[I]$ the direct sum of $K$-modules $(e A e / e I e) \oplus I$ with the multiplication

$$
(b, x) \cdot\left(b^{\prime}, x^{\prime}\right)=\left(b b^{\prime}, b x^{\prime}+x b^{\prime}+x x^{\prime}\right)
$$

for $b, b^{\prime} \in e A e / e I e$ and $x, x^{\prime} \in I$. Then $A[I]$ is an algebra with the identity $(e, 1-e)$ and, by identifying $x \in I$ with $(0, x) \in A[I]$, we may consider $I$ as an ideal of $A[I]$. The following fact has been proved in [7, Theorem 4.1].

Proposition 3.1. $A[I]$ is a self-injective algebra with a deforming ideal $I$, the Nakayama permutation of $A[I]$ is the same as the Nakayama permutation of $A$, and the algebras $A[I]$ and $A$ are socle equivalent.

Moreover, if $K$ is a field, we have proved in [8 Theorem 3] that the algebras $A[I]$ and $A$ are also stably equivalent. The aim of this section is to prove the following fact, needed in the proof of our main theorem.

Proposition 3.2. Let $A$ be a self-injective algebra with a deforming ideal $I, B=$ $A / I$ and let $e$ be a residual identity of $B$. Assume that $I e I=0$ and $e_{i} \neq e_{\nu(i)}$ for any primitive summand $e_{i}$ of $e$. Then the algebras $A[I]$ and $A$ are isomorphic.

Proof. We assume as before that $e=\sum_{i=1}^{m} e_{i}$ for $m \leq n$ and a set $\left\{e_{i} \mid 1 \leq i \leq n\right\}$ of primitive idempotents of $A$ with $1_{A}=\sum_{i=1}^{n} e_{i}$. Denote by $\bar{a}$ the residue class of $a \in e A e$ in $e A e / e I e$. Since by our assumption $\nu(i) \neq i$ for $i \in\{1, \ldots, m\}$, invoking Proposition 2.5 we have an algebra monomorphism $\phi: e A e / e I e \longrightarrow e A e$ such that $\phi(\bar{a})=\sum_{i=1}^{m} e_{i} a e_{B}^{(i)}$. We define a homomorphism $\Phi: A[I] \longrightarrow A$ of $K$-modules by

$$
\Phi(\bar{a}, x)=\phi(\bar{a})+x
$$

for $\bar{a} \in e A e / e I e$ and $x \in I$. We claim that $\Phi$ is an algebra isomorphism. Consider the following commutative diagram of canonical short exact sequences:

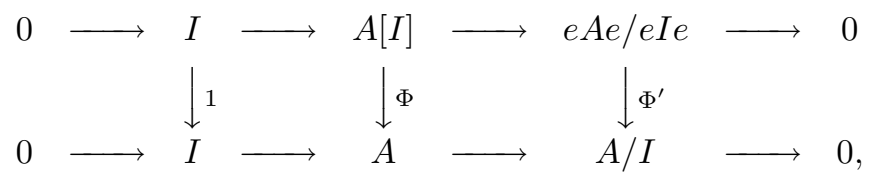

where 1 is the identity map and $\Phi^{\prime}$ is an isomorphism given by $\Phi^{\prime}(\bar{a})=\phi(\bar{a})+I$, for $\bar{a} \in e A e / e I e$. In order to prove that $\Phi$ is an algebra isomorphism, it suffices to show that $\Phi$ is an algebra homomorphism. Observe that

$$
\Phi\left(1_{A[I]}\right)=\Phi(\bar{e}, 1-e)=\phi(\bar{e})+(1-e)=e+(1-e)=1=1_{A} .
$$

Take $\bar{a}, \bar{b} \in e A e / e I e$ and $x, y \in I$. We shall prove that

$$
\Phi((\bar{a}, x)(\bar{b}, y))=\Phi(\bar{a}, x) \Phi(\bar{b}, y) .
$$


It follows from the definition of multiplication in $A[I]$ that

and

$$
\Phi((\bar{a}, x)(\bar{b}, y))=\Phi(\bar{a} \bar{b}, a y+x b+x y)=\phi(\bar{a} \bar{b})+(a y+x b)+x y
$$

$$
\Phi(\bar{a}, x) \Phi(\bar{b}, y)=(\phi(\bar{a})+x)(\phi(\bar{b})+y)=\phi(\bar{a}) \phi(\bar{b})+(\phi(\bar{a}) y+x \phi(\bar{b}))+x y .
$$

Hence, it suffices to show that $\phi(\bar{a}) y=a y$ and $x \phi(\bar{b})=x b$.

(a) $\phi(\bar{a}) y=a y$ for $a \in e A e$ and $y \in I$ :

In fact, $\phi(\bar{a}) y=\sum_{i=1}^{m} e_{i} a e_{B}^{(i)} y$, and

$$
a y=\left(\sum_{i=1}^{m} e_{i} a\right) y=\sum_{i} e_{i} a e_{B}^{(i)} y+\sum_{i} e_{i} a e_{I}^{(i)} y+\sum_{i} e_{i} a e_{\nu(i)} y .
$$

Here, $e_{i} a e_{I}^{(i)} y=0$ and $e_{i} a e_{\nu(i)} y=0$ by Lemma 2.2, because $e_{i} a e_{\nu(i)} \in \operatorname{rad} A$ by $\nu(i) \neq i$, and therefore $\phi(\bar{a}) y=a y$.

(b) $x \phi(\bar{b})=x b$ for $b \in e A e$ and $x \in I$ :

Since $e_{i} A e_{I}^{(i)} \subseteq e_{i} I$ by Lemma 2.1 we have $x e_{i} b e_{I}^{(i)} \in I e_{i} I \subseteq I e I$. Then $x e_{i} b e_{I}^{(i)}=0$ because $I e I=0$ by assumption. On the other hand, $e_{i} A e_{\nu(i)}=e_{i}(\operatorname{rad} A) e_{\nu(i)}$ by $\nu(i) \neq i$, and hence $e_{i} b e_{\nu(i)} \in I$ by (1) and (2) of Lemma 2.1. Hence $x e_{i} b e_{\nu(i)} \in$ $I e_{i} I \subseteq I e I=0$, and $x e_{i} b e_{\nu(i)}=0$. Thus,

$$
\begin{aligned}
x b & =\sum_{i=1}^{m} x e_{i} b e_{B}^{(i)}+\sum_{i} x e_{i} b e_{I}^{(i)}+\sum_{i} x e_{i} b e_{\nu(i)} \\
& =\sum_{i} x e_{i} b e_{B}^{(i)}=x \sum_{i} e_{i} b e_{B}^{(i)} \\
& =x \phi(\bar{b}) .
\end{aligned}
$$

Therefore, the algebras $A[I]$ and $A$ are isomorphic.

\section{Proof of the theorem}

We first prove the following general facts.

Lemma 4.1. Let $B$ be an algebra and $\varphi$ a positive automorphism of $\widehat{B}$ that fixes a finite subset of the object set of $\widehat{B}$. Then $\varphi$ is a rigid automorphism of $\widehat{B}$.

Proof. We write as before $\widehat{B}=\bigoplus_{k \in \mathbb{Z}}\left(B_{k} \oplus(D B)_{k}\right)$ with $B_{k}=B$ and $(D B)_{k}=D B$ for all $k \in \mathbb{Z}$, and identify the objects of $\widehat{B}$ (respectively, $B_{k}$ ) with the vertices of the ordinary quivers $Q(\widehat{B})$ (respectively, $Q\left(B_{k}\right)$ ). Since $\varphi$ is a positive automorphism of $\widehat{B}$, we may assume (without loss of generality) that $\varphi$ fixes a subset of $Q\left(B_{0}\right)$. In order to prove that $\varphi$ is rigid, it is enough to show that $\varphi\left(Q\left(B_{0}\right)\right)=Q\left(B_{0}\right)$. For this, since the quiver $Q\left(B_{0}\right)$ is connected, it suffices to show that $\varphi(\rightarrow S)=(\rightarrow S)$ and $\varphi(S \rightarrow)=(S \rightarrow)$ for any subset $S$ of $Q\left(B_{0}\right)$ with $\varphi(S)=S$, where $(\rightarrow S)$ and $(S \rightarrow)$ denote the sets of all predecessors of the vertices of $S$ and of all successors of the vertices of $S$ in $Q\left(B_{0}\right)$, respectively. Therefore, let $S$ be a subset of $Q\left(B_{0}\right)$ with $\varphi(S)=S$.

Now, let $x \rightarrow \cdots \rightarrow s$ be a path in $\mathcal{Q}\left(B_{0}\right)$ with $s \in S$. Then $\varphi(x) \rightarrow \cdots \rightarrow \varphi(s)$ is a path in $\mathcal{Q}(\widehat{B})$ and $\varphi(s) \in \mathcal{Q}\left(B_{0}\right)$, because $\varphi(S)=S$ by assumption. Since $\varphi$ is positive, $\varphi(x) \in \sum_{k \geq 0} \mathcal{Q}\left(B_{k}\right)$ and it follows that $\varphi(x)$ belongs to $\mathcal{Q}\left(B_{0}\right)$, because there is no path from $\mathcal{Q}\left(B_{k}\right)$ to $\mathcal{Q}\left(B_{0}\right)$ for any $k>0$. Thus $\varphi(\rightarrow S)=(\rightarrow S)$. 
Next, suppose that there is a path $s \rightarrow \cdots \rightarrow x$ in $\mathcal{Q}\left(B_{0}\right)$ for $s \in S$ with $\varphi(x) \notin \mathcal{Q}\left(B_{0}\right)$, that is, $\varphi(x) \in \mathcal{Q}\left(B_{k}\right)$ for some $k>0$. Since $S$ is finite and $Q(\widehat{B})$ is locally finite, the $\varphi$-orbit of $x$ is finite also. This implies that there is an integer $\ell$ such that $\varphi^{\ell}(x) \in \mathcal{Q}\left(B_{k^{\prime}}\right)$ for some $k^{\prime}>0$ and $\varphi^{\ell+1}(x) \in \mathcal{Q}\left(B_{0}\right)$. This, however, implies that $\varphi\left(\mathcal{Q}\left(B_{k^{\prime}}\right)\right) \cap \mathcal{Q}\left(B_{0}\right) \neq \emptyset$, which contradicts the positivity of $\varphi$. Thus we conclude that $\varphi(S \rightarrow)=(S \rightarrow)$.

Corollary 4.2. Let $B$ be an algebra and $\varphi$ a strictly positive automorphism of $\widehat{B}$. Then $\varphi$ acts freely on the objects of $\widehat{B}$.

Proof of the Theorem. Let $A$ be a self-injective algebra that is stably equivalent to a self-injective algebra of the form $\widehat{R} /\left(\psi \nu_{\widehat{R}}\right)$, where $R$ is a tilted algebra of type $\Delta$ (which is not a Dynkin quiver) and $\psi$ is a strictly positive automorphism of $\widehat{R}$. It follows from the proof of Theorem 1 in 8 that there are an ideal $I$ of $A$ and a residual identity $e=\sum_{i=1}^{m} e_{i}$ of $B=A / I$ such that $I e I=0, I e$ is an injective cogenerator in $\bmod B, B$ is a tilted algebra of type $\Delta$, and $A[I] \cong \widehat{B} /\left(\varphi \nu_{\widehat{B}}\right)$ for a positive automorphism $\varphi$ of $\widehat{B}$. Moreover, by [8, Theorem 3], the algebras $A$ and $A[I]$ are stably equivalent. Since by assumption $A$ is stably equivalent to $\widehat{R} /\left(\psi \nu_{\widehat{R}}\right)$, we conclude that $A[I]$ is stably equivalent to $\widehat{R} /\left(\psi \nu_{\widehat{R}}\right)$. Furthermore, since $\psi$ is a strictly positive automorphism of $\widehat{R}$, the number of simple modules of $\widehat{R} /(\psi \nu \widehat{R})$ is greater than the number of simple modules of $R$, which is the same as the number of vertices of $\Delta$. Applying now [8, Corollary] we conclude that the automorphism $\varphi$ is not rigid, and so is strictly positive. Hence, invoking Corollary 4.2 and Proposition 3.1, we obtain that $\nu(i) \neq i$ for the Nakayama permutation $\nu$ of $A$ and $i \in\{1, \ldots, m\}$. Since $B$ is a tilted algebra, the ordinary quiver $Q(B)$ of $B$ has no oriented cycles, and then the properties of $I$ stated above imply that $I$ is a deforming ideal of $A$. Therefore, applying Proposition 3.2. we obtain that $A$ and $A[I]$ are isomorphic, and so $A$ and $\widehat{B} /\left(\varphi \nu_{\widehat{B}}\right)$ are also isomorphic. This finishes the proof.

\section{ACKNOWLEDGEMENT}

The research was initiated during the visit of the second named author at the Nicholas Copernicus University (March 2002). The authors gratefully acknowledge support from the Foundation for Polish Science, the Polish Scientific Grant KBN No. 5 P03A 008 21, and the Japan Society for the Promotion of Science, Grant-inAid for Scientific Research (C) (2) No.12640014.

\section{REFERENCES}

[1] I. Assem, J. Nehring and A. Skowroński, Domestic trivial extensions of simply connected algebras, Tsukuba J. Math. 13 (1989), 31-72. MR 90j:16043

[2] M. Auslander, I. Reiten and S. O. Smalø, Representation Theory of Artin Algebras, Cambridge Studies in Advanced Mathematics 36, Cambridge, 1995. MR 96c:16015

[3] K. Erdmann, O. Kerner and A. Skowroński, Self-injective algebras of wild tilted type, J. Pure Appl. Algebra 149 (2000), 127-176. MR 2001g:16033

[4] P. Gabriel, The universal cover of a representation-finite algebra, In: Representations of Algebras, Lecture Notes in Math. 903, 68-105, Springer-Verlag, Berlin-Heidelberg-New York, 1981. MR 83f:16036

[5] D. Hughes and J. Waschbüsch, Trivial extensions of tilted algebras, Proc. London Math. Soc. 46 (1983), 347-364. MR 84m:16023 
[6] A. Skowroński, Self-injective algebras of polynomial growth, Math. Annalen 285 (1989), 177199. MR 90k:16024

[7] A. Skowroński and K. Yamagata, Socle deformations of self-injective algebras, Proc. London Math. Soc. 72 (1996), 545-566 MR 96m:16004

[8] A. Skowroński and K. Yamagata, Stable equivalence of self-injective algebras of tilted type, Arch. Math. (Basel) 70 (1998), 341-350. MR 99g:16017

[9] A. Skowroński and K. Yamagata, Galois coverings of self-injective algebras by repetitive algebras, Trans. Amer. Math. Soc. 351 (1999), 715-734. MR 99d:16008

[10] K. Yamagata, Representations of non-splittable extension algebras, J. Algebra 115 (1988), 32-45. MR 89d:16029

[11] K. Yamagata, Frobenius algebras, in: Handbook of Algebra 1, Vol.1, Elsevier, 1996, 841-887. MR 97k:16022

Faculty of Mathematics and Computer Science, Nicholas Copernicus University, Chopina 12/18, 87-100 Torún, Poland

E-mail address: skowron@mat.uni.torun.pl

Department of Mathematics, Tokyo University of Agriculture and Technology, NAKaCho 2-24-16, KoganeI, TOKYO 184-8588, JaPAN

E-mail address: yamagata@cc.tuat.ac.jp 